\title{
Prediction of metabolic syndrome by a high intake of energy-dense nutrient-poor snacks in Iranian children and adolescents
}

\author{
Golaleh Asghari', Emad Yuzbashian', Parvin Mirmiran², Zahra Bahadoran' and Fereidoun Azizi ${ }^{3}$
}

BACKGROUND: Our aim was to investigate the relationship between metabolic syndrome (MetS) and energy-dense nutrient-poor solid snacks intakes during 3.6 y of follow-up among children and adolescents.

METHOD: This population-based longitudinal study was conducted within the framework of the Tehran Lipid and Glucose Study, on 424 MetS free subjects, aged 6-18 y. Data on consumption of energy-dense nutrient-poor solid snacks, sweet and salty snacks, were collected at baseline (2006-2008). Logistic regression, adjusted for age, sex, total energy intake, physical activity, dietary fiber, family history of diabetes, and body mass index, was used to assess the relationship between snacks and incident MetS.

RESULTS: After adjustment for confounders, compared with the lowest quartile of total energy-dense nutrient-poor solid snacks, odds ratio of incident MetS in the highest quartile was 3.04 (95\% confidence interval (CI): 1.11-8.49); for sweet snacks this was 2.88 (95\% Cl: 1.00-8.32), and for salty ones it was 2.85 (95\% Cl: 1.09-7.43). The risk of incident hypertension in the highest, compared with the lowest quartile of total and salty snacks, was 2.99 (95\% Cl: 1.00-8.93) and 3.35 (95\% Cl: 1.1010.17), respectively.

CONCLUSION: Energy-dense nutrient-poor solid snacks, both salty and sweet, may have undesirable effects on the incidence of MetS, with salty snacks having the potential to increase risk of incident hypertension in children and adolescents as well.

C hildren and adolescents with metabolic syndrome (MetS) are at increased risk of many clinical conditions in adulthood, including chronic low-grade inflammation, oxidative stress, hypertension, dyslipidemia, polycystic ovary syndrome, nonalcoholic fatty liver disease, and certain forms of cancer (1). According to the International Diabetes Federation, the global prevalence of MetS in children is increasing (2), which has been attributed mainly to nutrition transition, i.e., a shift of dietary habits from the traditional, toward a more Western way of life (3). Major changes in snacking patterns have been observed, highlighting the marked increase in the contribution of snacks to total energy and saturated fat intake, in particular among children and adolescents (4).

Snacking patterns are usually identified based on the type of snack or the time when the snack was eaten (5). A recent review of current evidence on snack consumption and weight status reported data available to the controversial due to use of different definitions for snacking patterns and types of food that constituted snacks (4). According to their type, snack foods are categorized into two food groups, including energydense nutrient-poor (e.g., potato chips, chocolates, cakes, or biscuits) and low-energy high-nutrient (e.g., fruit or milk) snacks (6). Based on the snacking definition, energy-dense nutrient-poor snacks were associated with increased risk of obesity (7), whereas, in adolescents from Greece, no relationship was found between different levels of consumption and MetS and cardiovascular risk factors (8). Unlike previous studies, when snacking pattern was defined as any food consumed between regular meals, both (healthy and unhealthy) has been reported to be inversely associated with overweight and abdominal obesity (9).

Energy-dense nutrient-poor snacks, which are rich sources of simple sugars, sodium, and saturated and trans fatty acids, are associated with MetS in adults (10). Excessive amounts of sweeteners, such as sucrose in snacks, may contribute to increased energy intake, raise the glycemic indices, induce lipogenesis and $\beta$-cell dysfunction, and increase obesity and metabolic disorders $(11,12)$.

Although recent review has addressed the potential implications of snacking patterns for weight status (4), the contribution of energy-dense nutrient-poor snacks to metabolic abnormalities among child and adolescent populations in a longitudinal design study has not yet been fully elucidated. Therefore, this study was designed to describe the quantities of energy-dense nutrient-poor solid snacks among Iranian children and adolescents, aged 6-18 y, and to examine whether consumption of snacks, salty and sweet, was associated with incident MetS and its components after a mean follow up of $3.6 \mathrm{y}$.

\footnotetext{
${ }^{1}$ Nutrition and Endocrine Research Center, Research Institute for Endocrine Sciences, Shahid Beheshti University of Medical Sciences, Tehran, Iran; ${ }^{2}$ Department of Clinical Nutrition and Dietetics, Faculty of Nutrition Sciences and Food Technology, National Nutrition and Food Technology Research Institute, Shahid Beheshti University of Medical Sciences, Tehran, Iran; ${ }^{3}$ Endocrine Research Center, Research Institute for Endocrine Sciences, Shahid Beheshti University of Medical Sciences, Tehran, Iran. Correspondence: Parvin Mirmiran (mirmiran@endocrine.ac.ir)

Received 4 April 2015; accepted 16 October 2015; advance online publication 27 January 2016. doi:10.1038/pr.2015.270
} 


\section{Articles | Asghariet al.}

\section{RESULTS}

Mean (SD) age and body mass index (BMI) were 13.6 (3.7) y and $20.0(3.8) \mathrm{kg} / \mathrm{m}^{2}$, respectively, and mean intakes of sweet and salty snacks were $41.2 \pm 61.2$ and $20.3 \pm 31.4 \mathrm{~g} / \mathrm{d}$, respectively. Among 424 subjects (180 boys and 244 girls), with a mean follow-up of $>3.6$ y (range 2.31-4.87), there were 45 documented cases of MetS, respectively, with a cumulative incidence of $11 \%$. There was an eightfold difference in median intake of energy-dense nutrient-poor solid snacks between the highest and lowest quartiles of the study population (12 vs. $104 \mathrm{~g} / \mathrm{d}$ for total snacks). The contribution of energy-dense nutrient-poor solid snacks to total energy intake was $15 \%$ with a mean and median of 61.7 and $39.8 \mathrm{~g} / \mathrm{d}$, respectively. Baseline characteristics, except for age, were not significantly different between subjects followed up and those missed to followup (data not shown). The mean (SD) age of subjects missed to follow-up was higher than those followed $(14.9 \pm 3.4$ vs. $13.6 \pm 3.6 \mathrm{y}, P<0.05)$.

Characteristics of study children and adolescent participants according to quartiles of energy-dense nutrient-poor snack consumption are shown in Table 1. Participants in higher snack quartiles were younger, had higher waist circumference (WC), systolic blood pressure (SBP), diastolic blood pressure (DBP), and fasting plasma glucose (FPG), and consumed more simple sugars, fructose, total fat, saturated fat, monounsaturated fatty acids, and energy. Consumption of carbohydrates, polyunsaturated fatty acids, and protein decreased by higher quartiles of snacks intake.

The risk of incident MetS and its components according to quartiles of energy-dense nutrient-poor solid snacks are given in Table 2. In the fully adjusted model, in comparison with the lowest quartile subjects, those in the highest quartile of

Table 1. Baseline characteristics of children and adolescents according to quartiles of total energy-dense nutrient-poor solid snacks consumption:Tehran Lipid and Glucose Study

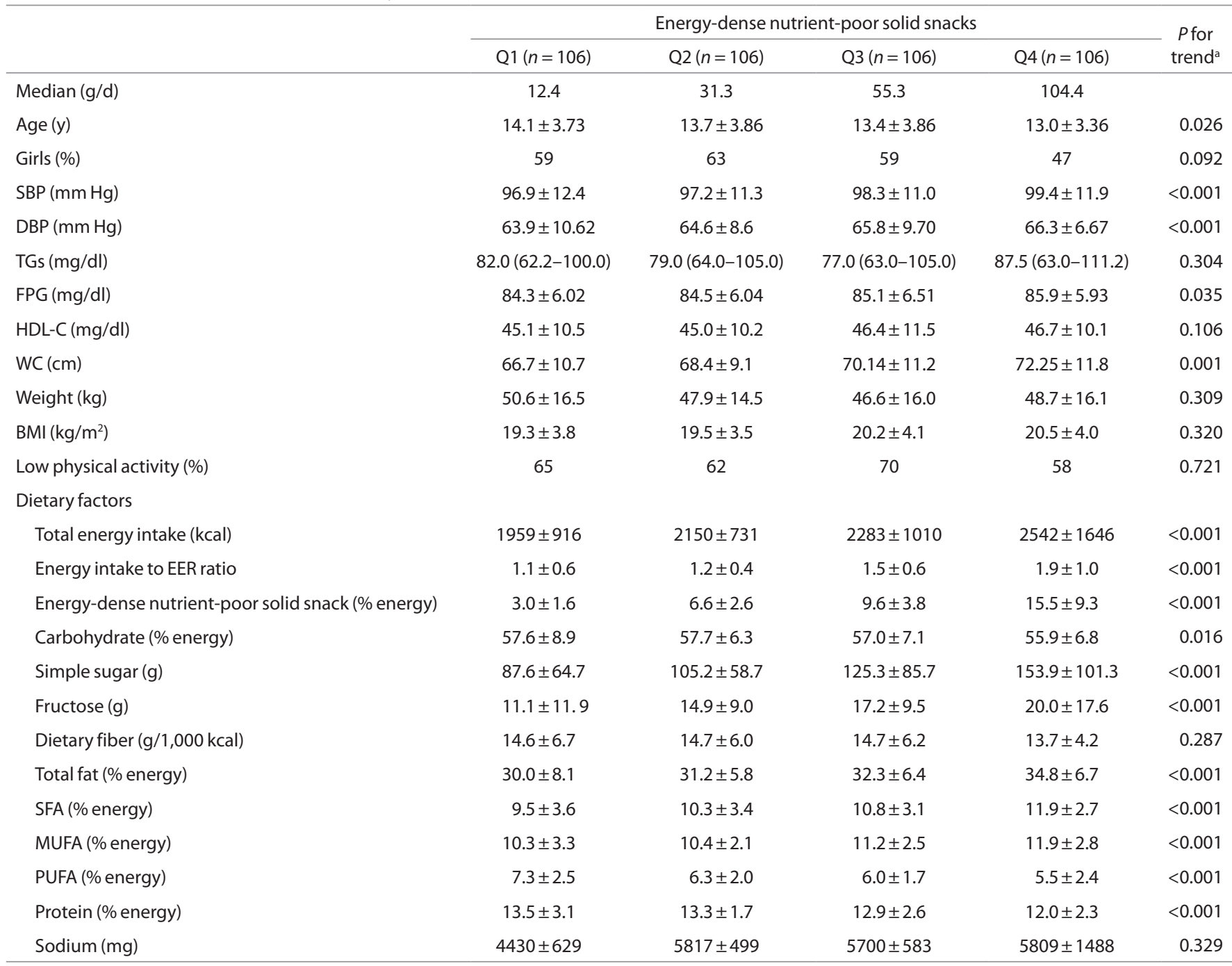

Data are represented as mean and SD or median (IQR 25-75) for continuous variables and as percentages for categorically distributed variables.

BMI, body mass index; DBP, diastolic blood pressure; EER, estimated energy requirement; FPG, fasting plasma glucose; HDL-C, high-density lipoprotein cholesterol; IQR, interquartile range; MUFA, monounsaturated fatty acid; PUFA, polyunsaturated fatty acid; SBP, systolic blood pressure; SFA, saturated fatty acid; TG, triglyceride; WC, waist circumference.

aLinear regression and chi-square test were used for continuous and categorical variables, respectively. 
Table 2. Multivariable-adjusted ORs ( $95 \% \mathrm{Cls}$ ) for incidence of MetS and its component by quartiles of energy-dense nutrient-poor solid snack intakes in children and adolescents during a 3.6-y follow-up

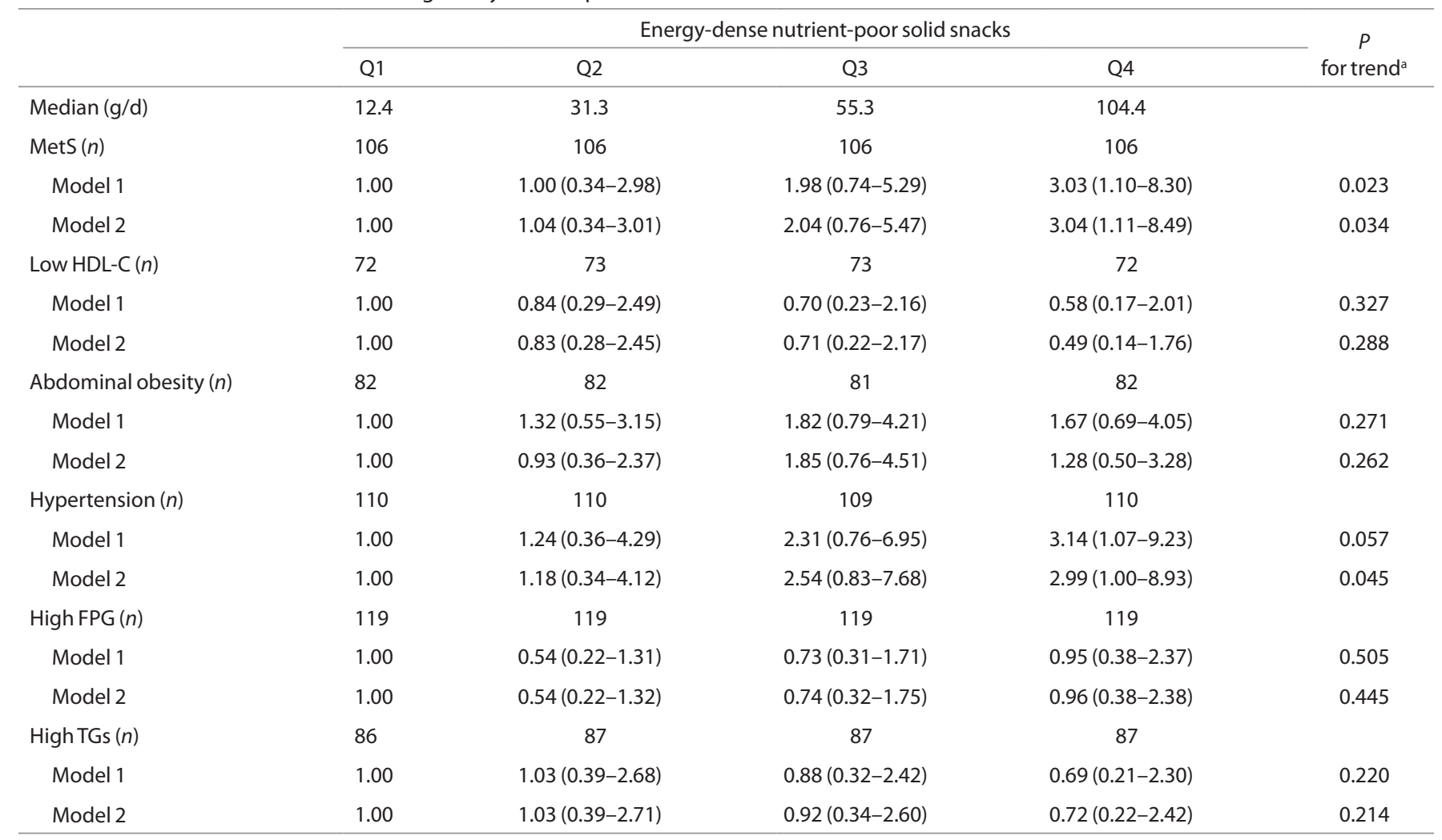

Model 1: Adjusted for age, sex, total energy intake, physical activity, dietary fiber, and family history of diabetes.

Model 2: Adjusted for model 1 variables plus BMI.

$\mathrm{BMI}$, body mass index; $\mathrm{Cl}$, confidence interval; $\mathrm{FPG}$, fasting plasma glucose; $\mathrm{HDL}-\mathrm{C}$, high-density lipoprotein cholesterol; MetS, metabolic syndrome; $\mathrm{OR}$, odds ratio; TG, triglyceride. aBased on logistic regression model using the median values for quartile of snack estimates as a continuous variable

snacks consumption had increased risk of incident MetS (odds ratio (OR): 3.04; $95 \%$ confidence interval (CI): 1.11-8.49, $P$ for trend $=0.034)$ and hypertension (OR: $2.99 ; 95 \%$ CI 1.00-8.93, $P$ for trend $=0.045$ ).

The ORs for incidence of MetS and its components according to quartiles of sweet and salty snack consumption are shown in Table 3; subjects in the highest quartile of sweet and salty snack consumption had increased risk of incident MetS, compared with those in the lowest, after adjustment for confounders ((OR: 2.88 ; 95\% CI: $1.00-8.32, P$ for trend $=0.024$ ) and (OR: 2.85 ; 95\% CI: $1.09-7.43, P$ for trend $=0.013$ ), respectively). Participants in the highest quartile of salty snacks showed an increased risk of incident hypertension (OR: 3.35; 95\% CI: 1.10-10.17; $P$ for trend $=0.046)$, in comparison with those in the lowest.

\section{DISCUSSION}

In this study, energy-dense nutrient-poor solid snack consumption, both sweet and salty, increased the risk of incident MetS, whereas intake of salty snacks was accompanied by increased risk of incident hypertension in children and adolescents, associations, which both remained significant after considering potential confounders, i.e., age, sex, total energy intake, physical activity, dietary fiber, family history of diabetes, and BMI.
Studies conducted in pediatrics investigating the relationship between dietary snacks and cardiometabolic risk factors are limited and their results are debatable $(7-9,13)$; some indicate that snacks are associated with unfavorable cardiovascular disease risk factors $(7,13)$, while others failed to establish any correlation or showed inverse correlations $(8,9)$. A positive association between blood pressure and the frequency of consumption of salty snack foods was found with $50 \%$ of daily salt intake from salty snacks (13). Middle-school Italian adolescents also showed higher prevalence of overweight and obesity with higher frequencies and calorie intakes of daily snacks (7), findings in contrast to those among Greek adolescents with an average consumption of $90 \mathrm{~g} / \mathrm{d}$ snacks, showing no significant association with MetS (8). In Australia, a study extracting dietary patterns of adolescents showed that participants with high scores of a Western dietary pattern, including sweet and salty snacks, were significantly associated with a greater risk of MetS (14). In our study, in particular salty snack consumption was positively associated with hypertension, even after controlling for BMI, results supported by observational and interventional studies showing that higher intakes of salt increase hypertension $(13,15)$.

The association between energy-dense nutrient-poor solid snack consumption and MetS in adolescents can be explained by several factors. Associations of snack intakes with MetS and 


\section{Articles | Asgharietal.}

Table 3. Multivariable-adjusted ORs ( $95 \% \mathrm{Cls}$ ) for incidence of MetS by quartiles of sweet and salty snacks intake in children and adolescents during a 3.6-y follow-up

\begin{tabular}{|c|c|c|c|c|c|}
\hline & \multicolumn{4}{|c|}{ Intakes } & \multirow{2}{*}{$\begin{array}{c}P \\
\text { for trend }\end{array}$} \\
\hline & Q1 & Q2 & Q3 & Q4 & \\
\hline Median sweet snacks (g/d) & 7 & 19.1 & 34.6 & 72.8 & \\
\hline Model 1 & 1.00 & $1.42(0.46-4.30)$ & $2.29(0.81-6.50)$ & $2.72(0.96-7.73)$ & 0.031 \\
\hline Model 2 & 1.00 & $1.42(0.46-4.32)$ & $2.35(0.82-6.73)$ & $2.88(1.00-8.32)$ & 0.024 \\
\hline Model 2 & 1.00 & $1.11(0.54-2.26)$ & $1.17(0.55-2.44)$ & $1.08(0.49-2.37)$ & 0.156 \\
\hline Abdominal obesity (n) & 82 & 82 & 81 & 82 & \\
\hline Model 1 & 1.00 & $0.57(0.26-1.26)$ & $1.03(0.50-2.13)$ & $0.56(0.25-1.27)$ & 0.215 \\
\hline Model 2 & 1.00 & $0.54(0.22-1.31)$ & $1.04(0.45-2.39)$ & $0.59(0.24-1.45)$ & 0.307 \\
\hline High FPG $(n)$ & 119 & 119 & 119 & 119 & \\
\hline Model 1 & 1.00 & $0.70(0.30-1.63)$ & $1.19(0.52-2.69)$ & $0.87(0.35-2.15)$ & 0.814 \\
\hline Model 2 & 1.00 & $0.70(0.30-1.62)$ & $1.18(0.52-2.68)$ & $0.87(0.35-2.16)$ & 0.836 \\
\hline High TGs (n) & 86 & 87 & 87 & 87 & \\
\hline Model 1 & 1.00 & $0.93(0.37-2.32)$ & $0.47(0.15-1.47)$ & $0.78(0.27-2.27)$ & 0.336 \\
\hline Model 2 & 1.00 & $0.92(0.37-2.28)$ & $0.49(0.16-1.52)$ & $0.87(0.29-2.58)$ & 0.520 \\
\hline Median Salty snacks (g/d) & 2.6 & 6.6 & 16.8 & 41.4 & \\
\hline MetS $(n)$ & 106 & 106 & 106 & 106 & \\
\hline Model 1 & 1.00 & $0.63(0.29-1.37)$ & $0.88(0.43-1.82)$ & $0.99(0.46-2.16)$ & 0.215 \\
\hline Model 2 & 1.00 & $0.61(0.26-1.46)$ & $0.78(0.34-1.76)$ & $0.72(0.30-1.74)$ & 0.307 \\
\hline Hypertension ( $n$ ) & 110 & 110 & 109 & 110 & \\
\hline Model 1 & 1.00 & $1.72(0.53-5.59)$ & $2.17(0.71-6.61)$ & $3.32(1.10-10.04)$ & 0.046 \\
\hline Model 2 & 1.00 & $1.84(0.56-5.99)$ & $2.27(0.74-6.96)$ & $3.35(1.10-10.17)$ & 0.046 \\
\hline High FPG $(n)$ & 119 & 119 & 119 & 119 & \\
\hline Model 1 & 1.00 & $0.47(0.19-1.15)$ & $0.89(0.40-1.94)$ & $1.16(0.50-2.66)$ & 0.200 \\
\hline Model 2 & 1.00 & $0.47(0.19-1.16)$ & $0.88(0.40-1.93)$ & $1.15(0.50-2.66)$ & 0.209 \\
\hline High TGs ( $n$ ) & 86 & 87 & 87 & 87 & \\
\hline Model 1 & 1.00 & $0.76(0.27-2.09)$ & $1.01(0.39-2.62)$ & $1.71(0.62-4.70)$ & 0.125 \\
\hline Model 2 & 1.00 & $0.78(0.28-2.17)$ & $1.02(0.39-2.68)$ & $1.76(0.63-4.90)$ & 0.117 \\
\hline
\end{tabular}

Model 1: Adjusted for age, sex, total energy intake, physical activity, dietary fiber, and family history of diabetes.

Model 2: Adjusted for variables in model 1 plus BMI.

Cl, confidence interval; FPG, fasting plasma glucose; HDL-C, high-density lipoprotein cholesterol; MetS, metabolic syndrome; OR, odds ratio; TG, triglyceride.

aBased on logistic regression model using the median values for quartile of sweet and salty snack estimates as a continuous variable.

its components were independent of energy intake and BMI, implying the potential of other snack constituents (fat, sugar, and salt) to predict MetS beyond BMI. Considering isocaloric levels, higher intakes of total simple sugars, fructose, total fat, and lower intakes of polyunsaturated fatty acids and protein may explain the observed associations, indicating that not only 
is the quantity of dietary intake important but quality of dietary intake and the source of calorie intake are also crucial. Evidence has confirmed the individual roles of different types of fat and carbohydrate on cardiometabolic risk factors $(16,17)$; He et al. (15) reported that British children, aged 4-18 y, showed an increase of $0.4 \mathrm{~mm} \mathrm{Hg}$ in SBP with each $1 \mathrm{~g} / \mathrm{d}$ intake of salt. A systematic review of environmental determinants of dietary behaviors, including attitudes, revealed that parental intake and parental education are undoubtedly associated with adolescents' intake (18). Physical, sociocultural, economic, and political factors in the school environment (school food policy and food prices) and neighborhood (availability and accessibility of foods in shops) are among other notable influential factors (18). Also, focusing on environmental level factors indicates that the major driving force for increases in noncommunicable diseases is a lifestyle encouraging unhealthy eating while overlooking physical activity $(19,20)$.

A study in the United States evaluating snacking trends in children (21) reported that during the last three decades, snacking behavior has increased in children of all age groups, constituting $27 \%$ of the daily caloric intake. In the current study, energy-dense nutrient-poor solid snacks comprised $>15 \%$ of total calorie intake, which was accompanied by higher intakes of total fat, simple sugars, and saturated fatty acids. A rise of energy-dense nutrient-poor solid snack consumption can have a number of reasons, such as environmental factors, including incorrect eating habits, family nutritional knowledge, TV publicizing, and their easy availability at school (22), all mainly attributed to the changes of dietary habits and a shift from the traditional toward a more Western way of life (23). In addition to their increased consumption, snacks are often also substituted for fruit and vegetable intakes, which are a part of low-energy high-nutrient diet (24).

During the cooking process of sweet snacks, large amounts of simple sugars are added, because of which these foods constitute rich sources of saturated and trans fatty acids; the high glycemic index of these sweet snacks hence causes a rise in blood sugar levels (25). Dietary glycemic index is associated with some cardiovascular disease risk factors, obesity, insulin resistance, and some components of MetS $(11,26)$. Saturated and trans fatty acids cause an increase in expression and secretion of inflammatory cytokines, such as interleukin-6 and C-reactive protein $(27,28)$. Persistent inflammation in the circulation disrupts metabolic homeostasis of the body and thus contributes to a family of disorders, including insulin resistance, glucose intolerance, hyperlipidemia, and hypertension, collectively known as metabolic syndrome (29).

Of our study limitations, the following need to be mentioned. First, data on the puberty status of the participants were not recorded, and we were not able to adjust puberty stage. Second, although research continues on determining a perfect site for WC assessment, for prediction of cardiovascular risk factors, WC was measured at the umbilicus level, however, almost all sites of WC measurements were accurate in predicting in general populations of children and adolescents $(30,31)$. Third, since household measures were not available for all food items in the food frequency questionnaire (FFQ), the standard US Department of Agriculture (USDA) serving sizes were applied as an alternative. Fourth, recall bias was also an inevitable problem when asking children to remember and report dietary intakes. Compared with the regular meals, children have more potential to forget the snacks they consumed may due to misreporting, food and drink items perceived as unhealthy are underreported more often than other foods, especially if the children are overweight (32-34). However, using well-trained interviewers, who are skilled in asking questions help subjects recall what they ate and also asking mothers about the type and quantity of meals and snacks, when children were unable to recall may help minimizing the memory limitations. Furthermore, we compensated for miss-reporting by using a valid and reliable FFQ and excluding over- and underreporters of energy intake. Again, we could not determine how many times per day the adolescents snacked, how much energy they consumed in each snack, when precisely they consumed snacks (during meals, e.g., potato chips and caramels, or between meals), and the setting, e.g., at home, at school, or in school neighborhoods, schools in which they snacked. The fifth limitation is that sugar sweetened beverages, including sugar sweetened carbonated soft drinks and fruit juice drinks, were not considered as energy-dense nutrient-poor snack in the analysis. Therefore, analysis focused only on energy-dense nutrient-poor solid snacks. Finally, despite controlling for various confounders in our analysis, residual confounding due to unknown or unmeasured confounders cannot be excluded. Our study had a prospective design and a population-based sample of participants and was adjusted for confounders such as lifestyle factors.

\section{Conclusion}

We found that high energy-dense nutrient-poor solid snack intakes, both salty and sweet, are associated with higher incidence of MetS and hypertension in children and adolescents, findings emphasizing the impact of consumption of large amounts and the composition of simple sugars, fructose, and fatty acids on the development of morbidity, independent of BMI.

\section{METHODS}

\section{Subjects}

Children and adolescents were selected from among participants of the Tehran Lipid and Glucose Study (TLGS), an ongoing prospective community-based investigation being conducted to determine the risk factors and prevent noncommunicable diseases by implementing and promoting healthy lifestyle (35). To summarize, 15,005 participants, aged $\geq 3 y$, residents under the coverage of three medical health centers in District No. 13 of Tehran, underwent baseline examinations between 1999 and 2002. Every 3 y, TLGS study participants are invited to come to the "Lipid and Glucose Research Unit" to update their data on demographic, lifestyle, biochemical, clinical, and dietary measurements using pretested questionnaires and standard protocols. The baseline survey was a cross-sectional study conducted from 1999 to 2001, and surveys 2 (2002-2005), 3 (2006-2008), and 4 (20092011) were prospective follow-up surveys.

In the third survey of the TLGS (2006-2008), from among 12,523 participants examined, 4,920 were randomly selected for completing dietary assessments, based on their age and sex; of these, 3,462 agreed to participate and completed the FFQ. Of participants who agreed to 
participate, $45.9,15.8,9.5$, and $18.3 \%$ were males, were $\leq 18$ y, were smokers, and had academic education, in comparison with 43.4, 18.9, 11.4 , and $16.5 \%$ of participants who refused to complete the FFQ, respectively.

For the current study, from among 3,462 participants with completed dietary assessment, 621 individuals, aged 6-18 y, were included. We excluded 29 subjects who did not have complete data on physical activity and anthropometric and biochemical variables. Furthermore, six subjects who were over- or underreported were also excluded. To define over- and underreports, the reported energy intake was divided by the estimated energy requirement (EER; calculated according to equations proposed by the Institute of Medicine (36), who were not within the $\pm 3 S D$ range (those in the top and bottom $1 \%$ of the energy intake to EER ratio). Depending on the outcome variables under analysis, subjects who had MetS $(n=69)$, hypertension $(n=53)$, high triglycerides (TGs; $n=168)$, low high-density lipoprotein cholesterol (HDL-C; $n=242)$, high fasting glucose $(n=12)$, or abdominal obesity $(n=145)$ were also excluded at baseline for determining the incidence of MetS and its components. Some individuals fell into more than one exclusion category. After a mean $3.6 \mathrm{y}$ of follow-up (2009-2011), eventually data of 424, 439, 347, 290, 476, and 327 participants remained for occurrence of MetS, hypertension, hypertriglyceridemia, low HDL-C, high FPG, and abdominal obesity, respectively (response rate was $86 \%$ ).

The design of this study was approved by the institutional ethics committee of the Research Institute for Endocrine Sciences, affiliated to the Shahid Beheshti University of Medical Sciences, and written informed consent was obtained from participants' parents.

\section{Measurements}

Using the Modifiable Activity Questionnaire, information on physical activity to calculate metabolic equivalent task minutes per week was collected. High reliability (97\%) and moderate validity (49\%) have been ascertained previously for the Persian translated Modifiable Activity Questionnaire in adolescents (37). Low level of physical activity was considered as metabolic equivalent task $<600 \mathrm{~min} / \mathrm{wk}$.

Weight was measured, while participants were minimally clothed without shoes, using digital scales (Seca 707, Seca, Hanover, MD; range $0.1-150 \mathrm{~kg}$ ) and was recorded to the nearest $100 \mathrm{~g}$. Height was measured in a standing position without shoes, using a stadiometer, with shoulders in normal alignment. BMI was calculated as weight $(\mathrm{kg})$ divided by square of height $\left(\mathrm{m}^{2}\right)$. WC was measured at the umbilicus, using a measuring tape without pressure to body surfaces and was recorded to the nearest $0.5 \mathrm{~cm}$.

To measure blood pressure, the participants remained seated for 15 min when a qualified physician, using a standard mercury sphygmomanometer with the cuff placed on the right arm, measured blood pressure twice, and the mean values were documented.

Blood samples, at baseline and follow-up, were drawn between 7:00 and 9:00 AM from all study participants after 12-14h of overnight fasting. All the blood analyses were done at the TLGS research laboratory on the day of blood collection. FPG was measured by the enzymatic colorimetric method using glucose oxidase. Serum HDL-C was measured after precipitation of the apolipoprotein B-containing lipoproteins with phosphotungstic acid, and serum TGs were assayed using an enzymatic colorimetric method with glycerol phosphate oxidase. These analyses were performed using commercial kits (Pars Azmoon, Tehran, Iran) and a Selectra 2 auto analyzer (Vital Scientific, Spankeren, The Netherlands). Inter- and intra-assay coefficients of variations at baseline were both $2.2 \%$ for FPG, 2.0 and $0.5 \%$ for HDLC, and 1.6 and $0.6 \%$ for TGs, respectively.

\section{Dietary Assessment}

A valid and reliable 168-item semiquantitative FFQ (38-40) was used by trained dietitians, during face-to-face interviews, to evaluate the usual dietary intakes of participants at baseline. Participants were asked to report their consumption frequency of food items during the previous year on a daily, weekly, or monthly basis, and data were then converted to mean daily intakes. Portion sizes of consumed foods, which were reported in household measures, were specified according to the USDA standard portion sizes (e.g., apple, 1 medium; bread, 1 slice; dairy, 1 cup) and were then converted to grams. When using USDA portion sizes was not possible, household measures (e.g., beans, 1 tablespoon; chicken meat, 1 leg or wing; rice, 1 large or small plate) were used alternatively.

To study the reproducibility of the FFQ, 132 subjects completed a 168-item FFQ (FFQ1 and FFQ2) twice with a 14-month interval between FFQ1 and FFQ2, and to assess the validity, 12 dietary recalls were collected (1 each month) over the 1-y interval. Age- and energyadjusted and deattenuated Spearman correlation coefficients used to assess validity of the FFQ for food groups were 0.44 and 0.37 in males and females, respectively; age- and energy-adjusted intraclass correlation coefficients, which reflect the reproducibility of food groups in the FFQ, were 0.52 and 0.57 in males and females, respectively (39).

As defined by Gregori et al. (5), snacks are categorized based on two main criteria: (i) the type of snack and (ii) the time when the snack was eaten (not consumed as part of breakfast, lunch, and dinner); in the current study, snacks were defined according to the first criteria, which included energy-dense nutrient-poor solid foods. Candies, chocolates, cookies, cakes, biscuits, confectionery, caramels, and traditional Iranian confectioneries, such as gaz, sohan, noghl, halva, Yazdi cakes, were all considered sweet snacks, and potato chips, popcorn, and puff (a corn snack or crisp coated with a mixture of cheese or cheese-flavored) were considered as salty ones. Total snack was the sum of sweet and salty snacks. These solid foods have high content of energy, added sugar, saturated fat, and salt. The association between sugar sweetened beverages and MetS among children and adolescents was investigated in another study, published recently (41). Sugar sweetened beverages were not included in snack consumption; nor were foods with high nutritional value, such as fruits, vegetables, and dairy products, considered as snacks.

\section{Definitions}

In children and adolescents, MetS was defined according to definition proposed by Cook et al. (42) as three or more of the following: WC $\geq$ 90th percentile for age and sex, according to national reference curves (43); SBP and DBP $\geq 90$ th percentile for sex, age, and height, from the National Heart, Lung, and Blood Institute's recommended cutoff points (44); FPG $\geq 100 \mathrm{mg} / \mathrm{dl}$, according to the recommendations of American Diabetes Association (45); fasting TGs $\geq 110 \mathrm{mg} / \mathrm{dl}$; and HDL-C $<40 \mathrm{mg} / \mathrm{dl}$. In subjects, aged $>18 \mathrm{y}$ after follow-up, MetS was defined according to the joint interim statement (46) as the presence of any three of five risk factors of the following: (i) Abdominal obesity for women $W C \geq 91 \mathrm{~cm}$ and for men $W C \geq 89 \mathrm{~cm}$, according to Iranians cutoff point (47); (ii) FPG $\geq 100 \mathrm{mg} / \mathrm{dl}$ or drug treatment; (iii) fasting TGs $\geq 150 \mathrm{mg} / \mathrm{dl}$ or drug treatment; (iv) fasting HDL-C $<$ $50 \mathrm{mg} / \mathrm{dl}$ for women and $<40 \mathrm{mg} / \mathrm{dl}$ for men or drug treatment; and (v) high blood pressure defined as $\mathrm{SBP} \geq 130 \mathrm{~mm} \mathrm{Hg}$ or $\mathrm{DBP} \geq 85 \mathrm{~mm}$ $\mathrm{Hg}$, or antihypertensive drug treatment.

\section{Statistical Analysis}

EER was calculated according to the equation proposed by Institute of Medicine (36), as follows:

For boys with BMIs between the 5th and 85th percentiles:

$$
\begin{aligned}
\text { EER }= & 88.5-61.9 \times \text { age }(y) \times \text { physical activity }+ \\
& {[26.7 \times \text { weight }(\mathrm{kg})+903 \times \text { height }(\mathrm{m})]+25 }
\end{aligned}
$$

For girls with BMIs between the 5th and 85th percentiles:

$$
\begin{aligned}
\text { EER }= & 135.3-30.8 \times \text { age }(\mathrm{y}) \times \text { physical activity }+ \\
& {[10 \times \text { weight }(\mathrm{kg})+934 \times \text { height }(\mathrm{m})]+25 }
\end{aligned}
$$

For boys with BMIs $>$ 85th percentile:

$$
\begin{aligned}
\text { EER }= & 114-50.9 \times \text { age }(\mathrm{y}) \times \text { physical activity }+ \\
& {[19.5 \times \text { weight }(\mathrm{kg})+1,161.4 \times \text { height }(\mathrm{m})] }
\end{aligned}
$$

For girls with BMIs > 85th percentile:

$$
\begin{aligned}
\text { EER }= & 389-41.2 \times \text { age }(\mathrm{y}) \times \text { physical activity }+ \\
& {[15 \times \text { weight }(\mathrm{kg})+701.6 \times \text { height }(\mathrm{m})] }
\end{aligned}
$$


Characteristics of subjects at baseline, according to quartiles of energy-dense nutrient-poor solid snack intakes, were expressed as mean and SD or median and interquartile range for continuous variables and as percentages for categorical variables. To investigate trend of variables according to the quartiles, linear regression and chi-square test were used for continuous and categorical variables, respectively. We analyzed the data of both the cohort that provided follow-up assessments and that of those lost at baseline. There were no significant differences in mean baseline anthropometric measurements and biochemical assessments.

Energy-dense nutrient-poor solid snacks, both sweet and salty, were divided into quartiles. Multiple logistic regression models were used to examine the association between MetS and its components in each quartile of snacks consumption. The OR and 95\% CIs for the incidence of the MetS were calculated. The initial multivariable models were adjusted for age, sex, total energy intake, physical activity, dietary fiber, and family history of diabetes. Multivariable models were further adjusted for BMI. Although categories of snacks intake did not differ significantly with respect to sex and dietary fiber, we adjusted these potential confounding factors, because they significantly altered the risk estimates. Also for consistency to facilitate comparison with previous studies, these variables were adjusted.

Tests of linear trend were conducted by assigning the medians of intakes in quartiles treated as a continuous variable. Statistical analysis was performed using the Statistical Package for Social Sciences (version 15.0; SPSS, Chicago IL).

\section{ACKNOWLEDGMENTS}

The authors thank the participants in the Tehran Lipid and Glucose Study for their enthusiastic support and the staff of the Tehran Lipid and Glucose Study Unit of the Research Institute for Endocrine Sciences for their valuable help. The authors also acknowledge Niloofar Shiva for critical editing of English grammar and syntax of the manuscript.

\section{STATEMENT OF FINANCIAL SUPPORT}

This study was supported by Grant 098 from the Research Institute for Endocrine Sciences, Shahid Beheshti University of Medical Sciences of Iran (Tehran, Iran). The funders had no role in study design, data collection and analysis, decision to publish, or preparation of the manuscript.

Disclosure: None of the authors had any personal or financial conflicts of interest.

\section{REFERENCES}

1. Cornier MA, Dabelea D, Hernandez TL, et al. The metabolic syndrome. Endocr Rev 2008;29:777-822.

2. Zimmet P, Alberti KG, Kaufman F, et al.; IDF Consensus Group. The metabolic syndrome in children and adolescents-an IDF consensus report. Pediatr Diabetes 2007;8:299-306.

3. Ghassemi H, Harrison G, Mohammad K. An accelerated nutrition transition in Iran. Public Health Nutr 2002;5:149-55.

4. Larson N, Story M. A review of snacking patterns among children and adolescents: what are the implications of snacking for weight status? Child Obes 2013;9:104-15.

5. Gregori D, Foltran F, Ghidina M, Berchialla P. Understanding the influence of the snack definition on the association between snacking and obesity: a review. Int J Food Sci Nutr 2011;62:270-5.

6. Gevers DW, Kremers SP, de Vries NK, van Assema P. Intake of energydense snack foods and drinks among Dutch children aged 7-12 years: how many, how much, when, where and which? Public Health Nutr 2015:1-10.

7. Bo S, De Carli L, Venco E, et al. Impact of snacking pattern on overweight and obesity risk in a cohort of 11- to 13-year-old adolescents. J Pediatr Gastroenterol Nutr 2014;59:465-71.

8. Vergetaki A, Linardakis M, Papadaki A, Kafatos A. Presence of metabolic syndrome and cardiovascular risk factors in adolescents and University students in Crete (Greece), according to different levels of snack consumption. Appetite 2011;57:278-85.

9. Keast DR, Nicklas TA, O'Neil CE. Snacking is associated with reduced risk of overweight and reduced abdominal obesity in adolescents: National
Health and Nutrition Examination Survey (NHANES) 1999-2004. Am J Clin Nutr 2010;92:428-35.

10. Mirmiran P, Bahadoran Z, Delshad H, Azizi F. Effects of energy-dense nutrient-poor snacks on the incidence of metabolic syndrome: a prospective approach in Tehran Lipid and Glucose Study. Nutrition 2014;30: 538-43.

11. Hosseinpour-Niazi S, Sohrab G, Asghari G, Mirmiran P, Moslehi N, Azizi F. Dietary glycemic index, glycemic load, and cardiovascular disease risk factors: Tehran Lipid and Glucose Study. Arch Iran Med 2013;16: 401-7.

12. Patterson E, Wärnberg J, Kearney J, Sjöström M. Sources of saturated fat and sucrose in the diets of Swedish children and adolescents in the European Youth Heart Study: strategies for improving intakes. Public Health Nutr 2010;13:1955-64.

13. Ponzo V, Ganzit GP, Soldati L, et al. Blood pressure and sodium intake from snacks in adolescents. Eur J Clin Nutr 2015;69:681-6.

14. Ambrosini GL, Huang RC, Mori TA, et al. Dietary patterns and markers for the metabolic syndrome in Australian adolescents. Nutr Metab Cardiovasc Dis 2010;20:274-83.

15. He FJ, Marrero NM, Macgregor GA. Salt and blood pressure in children and adolescents. J Hum Hypertens 2008;22:4-11.

16. Kuipers RS, de Graaf DJ, Luxwolda MF, Muskiet MH, Dijck-Brouwer DA, Muskiet FA. Saturated fat, carbohydrates and cardiovascular disease. Neth J Med 2011;69:372-8.

17. Schwab U, Lauritzen L, Tholstrup T, et al. Effect of the amount and type of dietary fat on cardiometabolic risk factors and risk of developing type 2 diabetes, cardiovascular diseases, and cancer: a systematic review. Food Nutr Res 2014;58.

18. van der Horst K, Oenema A, Ferreira I, et al. A systematic review of environmental correlates of obesity-related dietary behaviors in youth. Health Educ Res 2007;22:203-26.

19. French SA, Story M, Jeffery RW. Environmental influences on eating and physical activity. Annu Rev Public Health 2001;22:309-35.

20. Swinburn BA, Caterson I, Seidell JC, James WP. Diet, nutrition and the prevention of excess weight gain and obesity. Public Health Nutr 2004;7: 123-46.

21. Piernas C, Popkin BM. Trends in snacking among U.S. children. Health Aff (Millwood) 2010;29:398-404.

22. Weker H. [Simple obesity in children. A study on the role of nutritional factors]. Med Wieku Rozwoj 2006;10:3-191.

23. Kafatos A, Kouroumalis I, Vlachonikolis I, Theodorou C, Labadarios D. Coronary-heart-disease risk-factor status of the Cretan urban population in the 1980s. Am J Clin Nutr 1991;54:591-8.

24. Cohen DA, Sturm R, Scott M, Farley TA, Bluthenthal R. Not enough fruit and vegetables or too many cookies, candies, salty snacks, and soft drinks? Public Health Rep 2010;125:88-95.

25. Jenkins DJ, Wolever TM, Taylor RH, et al. Glycemic index of foods: a physiological basis for carbohydrate exchange. Am J Clin Nutr 1981;34:362-6.

26. Rouhani MH, Salehi-Abargouei A, Azadbakht L. Effect of glycemic index and glycemic load on energy intake in children. Nutrition 2013;29:1100-5.

27. Mozaffarian D, Pischon T, Hankinson SE, et al. Dietary intake of trans fatty acids and systemic inflammation in women. Am J Clin Nutr 2004;79: 606-12.

28. Baer DJ, Judd JT, Clevidence BA, Tracy RP. Dietary fatty acids affect plasma markers of inflammation in healthy men fed controlled diets: a randomized crossover study. Am J Clin Nutr 2004;79:969-73.

29. Purkayastha S, Cai D. Neuroinflammatory basis of metabolic syndrome. Mol Metab 2013;2:356-63.

30. Andaki AC, Tinoco AL, Mendes EL, Andaki Júnior R, Hills AP, Amorim PR. Different waist circumference measurements and prediction of cardiovascular risk factors and metabolic syndrome in children. Obes Res Clin Pract 2012;6:e91-e174.

31. Koot BG, Westerhout R, Bohte AE, et al. Ultrasonography is not more reliable than anthropometry for assessing visceral fat in obese children. Pediatr Obes 2014;9:443-7.

32. Vance VA, Woodruff SJ, McCargar LJ, Husted J, Hanning RM. Self-reported dietary energy intake of normal weight, overweight and obese adolescents. Public Health Nutr 2009;12:222-7. 


\section{Articles | Asghariet al.}

33. Johansson L, Solvoll K, Bjørneboe GE, Drevon CA. Under- and overreporting of energy intake related to weight status and lifestyle in a nationwide sample. Am J Clin Nutr 1998;68:266-74.

34. Olafsdottir AS, Thorsdottir I, Gunnarsdottir I, Thorgeirsdottir H, Steingrimsdottir L. Comparison of women's diet assessed by FFQs and 24-hour recalls with and without underreporters: associations with biomarkers. Ann Nutr Metab 2006;50:450-60.

35. Azizi F, Ghanbarian A, Momenan AA, et al.; Tehran Lipid and Glucose Study Group. Prevention of non-communicable disease in a population in nutrition transition: Tehran Lipid and Glucose Study phase II. Trials 2009;10:5.

36. Panel on Macronutrients, Panel on the Definition of Dietary Fiber, Subcommittee on Upper Reference Levels of Nutrients, Subcommittee on Interpretation and Uses of Dietary Reference Intakes, and the Standing Committee on the Scientific Evaluation of Dietary Reference Intakes. Dietary Reference Intakes for Energy, Carbohydrate, Fiber, Fat, Fatty Acids, Cholesterol, Protein and Amino Acids. Washington, DC: Institute of Medicine, Food and Nutrition Board. National Academies Press, 2005.

37. Delshad M, Ghanbarian A, Ghaleh NR, Amirshekari G, Askari S, Azizi F. Reliability and validity of the modifiable activity questionnaire for an Iranian urban adolescent population. Int J Prev Med 2015;6:3.

38. Mirmiran P, Esfahani FH, Mehrabi Y, Hedayati M, Azizi F. Reliability and relative validity of an FFQ for nutrients in the Tehran lipid and glucose study. Public Health Nutr 2010;13:654-62.

39. Esfahani FH, Asghari G, Mirmiran P, Azizi F. Reproducibility and relative validity of food group intake in a food frequency questionnaire developed for the Tehran Lipid and Glucose Study. J Epidemiol 2010;20:150-8.

40. Asghari G, Rezazadeh A, Hosseini-Esfahani F, Mehrabi Y, Mirmiran P, Azizi F. Reliability, comparative validity and stability of dietary patterns derived from an FFQ in the Tehran Lipid and Glucose Study. Br J Nutr 2012;108:1109-17.

41. Mirmiran P, Yuzbashian E, Asghari G, Hosseinpour-Niazi S, Azizi F. Consumption of sugar sweetened beverage is associated with incidence of metabolic syndrome in Tehranian children and adolescents. Nutr Metab (Lond) 2015;12:25.

42. Cook S, Weitzman M, Auinger P, Nguyen M, Dietz WH. Prevalence of a metabolic syndrome phenotype in adolescents: findings from the third National Health and Nutrition Examination Survey, 1988-1994. Arch Pediatr Adolesc Med 2003;157:821-7.

43. Kelishadi R, Gouya MM, Ardalan G, et al.; CASPIAN Study Group. First reference curves of waist and hip circumferences in an Asian population of youths: CASPIAN study. J Trop Pediatr 2007;53:158-64.

44. National High Blood Pressure Education Program Working Group on High Blood Pressure in Children and Adolescents. The fourth report on the diagnosis, evaluation, and treatment of high blood pressure in children and adolescents. Pediatrics 2004;114:555-76.

45. Genuth S, Alberti KG, Bennett P, et al.; Expert Committee on the Diagnosis and Classification of Diabetes Mellitus. Follow-up report on the diagnosis of diabetes mellitus. Diabetes Care 2003;26:3160-7.

46. Alberti KG, Eckel RH, Grundy SM, et al.; International Diabetes Federation Task Force on Epidemiology and Prevention; Hational Heart, Lung, and Blood Institute; American Heart Association; World Heart Federation; International Atherosclerosis Society; International Association for the Study of Obesity. Harmonizing the metabolic syndrome: a joint interim statement of the International Diabetes Federation Task Force on Epidemiology and Prevention; National Heart, Lung, and Blood Institute; American Heart Association; World Heart Federation; International Atherosclerosis Society; and International Association for the Study of Obesity. Circulation 2009;120:1640-5.

47. Delavari A, Forouzanfar MH, Alikhani S, Sharifian A, Kelishadi R. First nationwide study of the prevalence of the metabolic syndrome and optimal cutoff points of waist circumference in the Middle East: the national survey of risk factors for noncommunicable diseases of Iran. Diabetes Care 2009;32:1092-7. 